\title{
Deformation of algebraic cycle classes in characteristic zero
}

\author{
Spencer Bloch, Hélène Esnault and Moritz Kerz
}

\begin{abstract}
We study a formal deformation problem for rational algebraic cycle classes motivated by Grothendieck's variational Hodge conjecture. We argue that there is a close connection between the existence of a Chow-Künneth decomposition and the existence of expected deformations of cycles. This observation applies in particular to abelian schemes.
\end{abstract}

\section{Introduction}

Let $K$ be a field of characteristic 0 . Let $Z / K$ be a smooth projective variety of dimension $d$ and let $i$ be an integer. The following Chow-Künneth property is expected to hold in general as part of conjectures of Grothendieck, Beilinson and Murre, see [Jan94, Sec. 5].

Property $(\mathbf{C K})_{Z}^{i}$ : There is an idempotent correspondence $\pi^{i} \in \mathrm{CH}^{d}\left(Z \times_{K} Z\right)_{\mathbb{Q}}$ such that on $H_{\mathrm{dR}}^{*}(Z / K)$ the correspondence $\pi^{i}$ acts as the projection to $H_{\mathrm{dR}}^{i}(Z / K)$.

Remark 1.1. An important and well-understood example is the case of an abelian variety $A / K$. In this case we know $(\mathbf{C K})_{A}^{i}$ for all integers $i$, see for instance [DM91].

Set $S=\operatorname{Spec} k[[t]]$, where $k$ is a field of characteristic 0 . Let $X / S$ be a smooth projective scheme. Let $\eta$ be the generic point of $S$, and let $K=k(\eta)$. Denote by $X_{n}$ the scheme $X \times_{S} S_{n}$ with $S_{n}=\operatorname{Spec} k[t] /\left(t^{n}\right)$.

There is a Chern character ring homomorphism to the de Rham cohomology

$$
\operatorname{ch}: K_{0}\left(X_{1}\right) \rightarrow H_{\mathrm{dR}}^{*}\left(X_{1} / k\right) .
$$

Determining the image of the Chern character is an important problem. In this note we consider an 'infinitesimal' analogue of this problem along the thickening $X_{1} \hookrightarrow X_{n}$.

By $\nabla: H_{\mathrm{dR}}^{*}(X / S) \rightarrow H_{\mathrm{dR}}^{*}(X / S)$ we denote derivation along the parameter $t$ with respect to the Gauss-Manin connection and by $H_{\mathrm{dR}}^{*}(X / S)^{\nabla}$ we denote the kernel of $\nabla$. Solving a formal differential equation we see that the canonical map

$$
\Phi: H_{\mathrm{dR}}^{*}(X / S)^{\nabla \stackrel{\sim}{\rightarrow}} H_{\mathrm{dR}}^{*}\left(X_{1} / k\right)
$$

is an isomorphism [Kat70, Prop. 8.9].

Received 22 October 2013, accepted in final form 13 February 2014.

2010 Mathematics Subject Classification 14C25, 14C35

Keywords: deformations of algebraic cycles, variational Hodge conjecture

This journal is (C) Foundation Compositio Mathematica 2014. This article is distributed with Open Access under the terms of the Creative Commons Attribution Non-Commercial License, which permits non-commercial reuse, distribution, and reproduction in any medium, provided that the original work is properly cited. For commercial re-use, please contact the Foundation Compositio Mathematica.

The second author is supported by the Einstein Foundation, the ERC Advanced Grant 226257, the third author by the DFG Emmy-Noether Nachwuchsgruppe "Arithmetik über endlich erzeugten Körpern" . 


\section{Deformation of CYCle ClASSES}

We denote by $F^{r} H_{\mathrm{dR}}^{i} \subset H_{\mathrm{dR}}^{i}$ the Hodge filtration on the de Rham cohomology (of smooth projective schemes). Our main theorem is as follows.

TheOrem 1.2. Assume that for the scheme $X / S$ as above the property $(\mathbf{C K})_{X_{\eta}}^{i}$ holds for all even $i \in \mathbb{Z}$. Then for $\xi_{1} \in K_{0}\left(X_{1}\right)_{\mathbb{Q}}$ the following are equivalent:

(i) $\Phi^{-1} \circ \operatorname{ch}\left(\xi_{1}\right) \in \bigoplus_{i} H_{\mathrm{dR}}^{2 i}(X / S)^{\nabla} \cap F^{i} H_{\mathrm{dR}}^{2 i}(X / S)$;

(ii) there is an element $\hat{\xi} \in\left(\lim _{n} K_{0}\left(X_{n}\right)\right) \otimes \mathbb{Q}$ such that

$$
\operatorname{ch}\left(\left.\hat{\xi}\right|_{X_{1}}\right)=\operatorname{ch}\left(\xi_{1}\right) \in H_{\mathrm{dR}}^{*}\left(X_{1} / k\right) .
$$

A preliminary version of Theorem 1.2 for cohomological Chow groups is shown in Section 5, see Theorem 5.2. The central new ingredient of our proof is studying a ring of correspondences for the nonreduced scheme $X_{n}$. Property $(\mathbf{C K})_{X_{\eta}}^{i}$ will guarantee that there are enough such correspondences in order to kill the influence of absolute differential forms of $k$ on the deformation behavior. In fact, for $\Omega_{k / \mathbb{Q}}^{1}=0$ the whole deformation problem is much easier, see Remark 1.3. The proof of Theorem 1.2 is completed via a Chern character isomorphism relying on Zariski descent for algebraic $K$-theory, see Section 6.

Remark 1.3. In case $k$ is algebraic over $\mathbb{Q}$ we also deduce without assuming $(\mathbf{C K})_{X_{\eta}}^{i}$ that conditions (i) and (ii) are equivalent and they are also equivalent to

(ii') there is an element $\hat{\xi} \in\left(\lim _{n} K_{0}\left(X_{n}\right)\right) \otimes \mathbb{Q}$ such that $\left.\hat{\xi}\right|_{X_{1}}=\xi_{1}$.

See related work [GG04], [PR13], [Mor13a]. However, our methods do not show that for general fields $k$ condition (ii') is equivalent to condition (ii) and we do not see a good reason to expect this.

Theorem 1.2 is motivated by a conjecture of Grothendieck [Gro66, p. 103], which is today called the variational Hodge conjecture. See Appendix A for his original global formulation. In this appendix it is shown that the latter is equivalent to the following "infinitesimal" conjecture.

Conjecture 1.4 (Infinitesimal Hodge). Statement (i) of Theorem 1.2 is equivalent to

(iii) there is an element $\xi \in K_{0}(X)_{\mathbb{Q}}$ such that

$$
\operatorname{ch}\left(\left.\xi\right|_{X_{1}}\right)=\operatorname{ch}\left(\xi_{1}\right) \in H_{\mathrm{dR}}^{*}\left(X_{1} / k\right) .
$$

One shows directly that (iii) $\Rightarrow$ (ii) $\Rightarrow(\mathrm{i})$ without assuming $(\mathbf{C K})_{X_{\eta}}^{i}$ for $i$ even. Conjecture 1.4 is particularly interesting for abelian schemes. Indeed it is known ([Abd94], [And96, Sec. 6]) that

Conjecture 1.4 for abelian schemes $X / S \Longrightarrow$ Hodge conjecture for abelian varieties.

As $(\mathbf{C K})_{X_{\eta}}^{i}$ is known for abelian varieties, see Remark 1.1, one can speculate about what is needed to deduce Conjecture 1.4 for abelian schemes from Theorem 1.2. In order to accomplish this one would have to solve an algebraization problem, namely one has to consider the question how far the map

$$
K_{0}(X) \rightarrow \underset{n}{\lim } K_{0}\left(X_{n}\right)
$$

is from being surjective (after tensoring with $\mathbb{Q}$ ). Recall that for line bundles the corresponding map

$$
\operatorname{Pic}(X) \rightarrow \underset{n}{\lim _{n}} \operatorname{Pic}\left(X_{n}\right)
$$




\section{Bloch, Esnault and Kerz}

is an isomorphism by formal existence [EGA3, III.5].

By considering a trivial deformation of an abelian surface we show in Appendix B that the map (1.3) cannot be surjective in general. For abelian schemes the counterexample leaves the following algebraization question open.

Question 1.5. Let $X / S$ be an abelian scheme and let $\ell>1$ be an integer. Is the map

$$
K_{0}(X)^{\psi^{\ell^{2}}-[\ell]^{*}} \rightarrow \underset{n}{\lim _{n}} K_{0}\left(X_{n}\right)^{\ell^{\ell^{2}}-[\ell]^{*}}
$$

surjective after tensoring with $\mathbb{Q}$ ?

Here $\psi^{\ell}$ is the $\ell$-th Adams operation [FL85] and [ $\left.\ell\right]: X \rightarrow X$ is multiplication by $\ell$. The upper index notation means that we take the kernel of the corresponding endomorphism.

From Theorem 1.2 we deduce the following result.

Corollary 1.6. A positive answer to Question 1.5 would imply the Hodge conjecture for abelian varieties.

\section{Milnor $K$-theory and differential forms}

Let $k$ be a field of characteristic 0 and write $S_{n}=\operatorname{Spec} k[t] /\left(t^{n}\right)$. Let $S=\operatorname{Spec} k[[t]]$, and let $X \rightarrow S$ be a smooth, separated scheme of finite type. Write $X_{n}=X \times_{S} S_{n}$. Write $\Omega_{X_{n}}^{r}, Z_{X_{n}}^{r}$ and $B_{X_{n}}^{r}$ for the Zariski sheaf of absolute $n$-forms, closed absolute $n$-forms and exact absolute $n$-forms on $X_{n}$, respectively. Let $\mathcal{K}_{*}^{\mathrm{M}}$ be the Milnor $K$-sheaf with respect to Zariski topology as studied in [Ker09].

Lemma 2.1. There is an exact sequence of Zariski sheaves

$$
0 \rightarrow \Omega_{X_{1}}^{r-1} \stackrel{a}{\rightarrow} Z_{X_{n}}^{r} \stackrel{f}{\rightarrow} Z_{X_{n-1}}^{r},
$$

where $a(\eta)=t^{n-1} d \eta+(n-1) t^{n-2} d t \wedge \eta$.

Proof. Note that $a$ is well defined because $t^{n}=t^{n-1} d t=0$ on $X_{n}$. Since

$$
\operatorname{ker}\left(\Omega_{X_{n}}^{r} \rightarrow \Omega_{X_{n-1}}^{r}\right)=t^{n-1} \Omega_{X_{n}}^{r}+t^{n-2} d t \wedge \Omega_{X_{n}}^{r-1},
$$

the assertion is clear.

Lemma 2.2. There is an exact sequence of Zariski sheaves

$$
\Omega_{X_{1}}^{r-1} \stackrel{b}{\rightarrow} \mathcal{K}_{r, X_{n}}^{\mathrm{M}} \rightarrow \mathcal{K}_{r, X_{n-1}}^{\mathrm{M}} \rightarrow 0 .
$$

Here $b\left(x d \log \left(y_{1}\right) \wedge \cdots \wedge d \log y_{r-1}\right)=\left\{1+x t^{n-1}, y_{1}, \ldots, y_{r-1}\right\}$.

Proof. We can present $\Omega_{X_{1}}^{1}$ with an exact sequence of $\mathcal{O}_{X_{1}}$-modules

$$
0 \rightarrow \mathcal{R} \rightarrow \mathcal{O}_{X_{1}} \otimes_{\mathbb{Z}} \mathcal{O}_{X_{1}}^{\times} \stackrel{u \otimes v \mapsto u d v / v}{\longrightarrow} \Omega_{X_{1}}^{1} \rightarrow 0
$$

Here $\mathcal{R}$ is the sub- $\mathcal{O}_{X_{1}}$-module under left multiplication with generators of the form $a \otimes a+b \otimes b-$ $(a+b) \otimes(a+b)$. It follows that $\Omega_{X_{1}}^{r-1}=\bigwedge_{\mathcal{O}_{X_{1}}}^{r-1} \Omega_{X_{1}}^{1}$ has a presentation of the form

$$
\mathcal{R} \otimes_{\mathbb{Z}} \bigwedge_{\mathbb{Z}}^{r-2} \mathcal{O}_{X_{1}}^{\times} \rightarrow \mathcal{O}_{X_{1}} \otimes_{\mathbb{Z}} \bigwedge_{\mathbb{Z}}^{r-1} \mathcal{O}_{X_{1}}^{\times} \rightarrow \Omega_{X_{1}}^{r-1} \rightarrow 0 .
$$


For $r=2$ the map

$$
\Omega_{X_{1}}^{1} \cong t^{n-1} \Omega_{X_{n}}^{1} \rightarrow \mathcal{K}_{2, X_{n}}^{\mathrm{M}}, \quad c d a / a \mapsto\left\{1+c t^{n-1}, a\right\}
$$

is well defined. This boils down to showing that

$$
\left\{1+a t^{n-1}, a\right\}+\left\{1+b t^{n-1}, b\right\}-\left\{1+(a+b) t^{n-1}, a+b\right\}=0
$$

in $\mathcal{K}_{2, X_{n}}$ for $a, b, a+b$ in $\mathcal{O}_{X_{1}}^{\times}$. See [Blo75, Sec. 2] for more details. Then from the presentation (2.5) we deduce that $b$ is well defined. The exactness of (2.3) is straightforward.

Proposition 2.3. The square

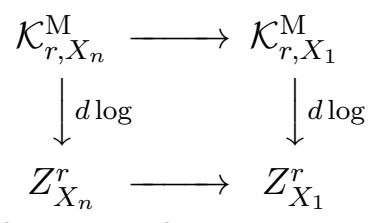

is cartesian and there is a morphism between short exact sequences

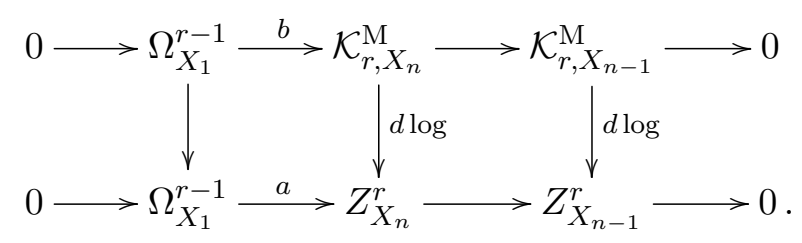

Proof. We have

$$
\mathcal{K}_{r, X_{1}}^{\mathrm{M}} \times Z_{X_{1}}^{r} Z_{X_{n}}^{r}=\mathcal{K}_{r, X_{1}}^{\mathrm{M}} \times Z_{X_{1}}^{r} Z_{X_{n-1}}^{r} \times Z_{X_{n-1}}^{r} Z_{X_{n}}^{r} .
$$

In order to prove the first statement by induction we are thus reduced to proving that the diagram

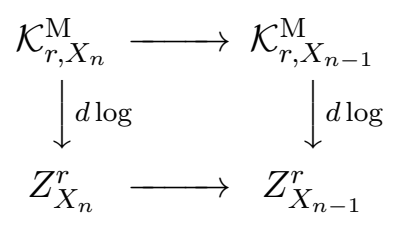

is cartesian. Plugging in Lemmas 2.1 and 2.2 yields

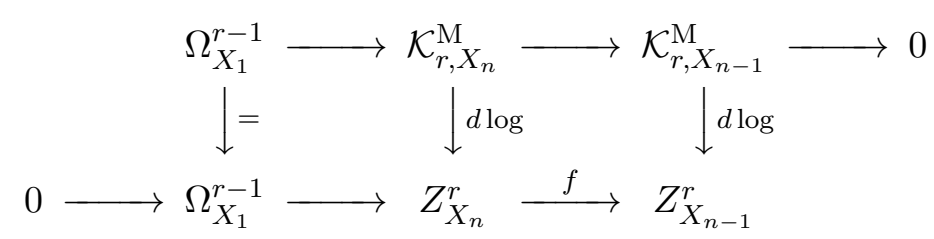

It follows that (2.10) is cartesian and that the upper row in (2.8) is exact as claimed. The exactness of the lower row of (2.8), that is, the surjectivity of $f$ in (2.11), follows from the commutative diagram of short exact sequences

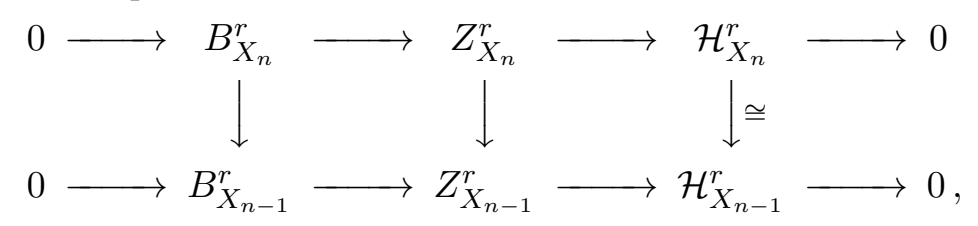

where $\mathcal{H}^{r}$ is the de Rham cohomology sheaf. Indeed, the right vertical map is an isomorphism by Lemma 2.4 and the left vertical map is obviously surjective.

LEMma 2.4. With notation as above, the map of complexes $\Omega_{X_{n}}^{*} \rightarrow \Omega_{X_{1}}^{*}$ is a quasi-isomorphism. 


\section{Bloch, Esnault and Kerz}

Proof. The assertion is local so we may assume that $X_{n}=\operatorname{Spec} A_{n}$ is affine. The algebra $A_{1}=A_{n} / t A_{n}$ is therefore smooth over $k$, so by the infinitesimal criterion for smoothness [EGA4, (17.1.1)], there exists a splitting of the surjection $A_{n} \rightarrow A_{1}$. It follows that we may write $X_{n} \cong X_{1} \times{ }_{k} S_{n}$. This implies that there is an isomorphism between differential graded algebras $\Omega_{X_{n}}^{*} \cong \Omega_{X_{1}}^{*} \otimes_{\mathbb{Q}} \Omega_{S_{n}}^{*}$. Since there is a short exact sequence

$$
0 \rightarrow t k[t] / t^{n} k[t] \stackrel{d}{\rightarrow} \Omega_{S_{n}}^{1} \rightarrow \Omega_{k / \mathbb{Q}}^{1} \rightarrow 0
$$

we deduce that $\Omega_{A_{n}}^{*} \rightarrow \Omega_{A_{0}}^{*}$ is a quasi-isomorphism as claimed.

\section{Local cohomology}

Let $X, S, X_{n}, S_{n}$ and $k$ be as in Section 2. One of the central techniques for proving our main Theorem 1.2 will be to study the coniveau complex for Milnor $K$-sheaves of $X_{n}$. A general reference for the coniveau complex is [Har66, Ch. IV].

Definition 3.1. For an arbitrary Zariski sheaf of abelian groups $\mathcal{F}$ on $X_{1}$, let us consider the coniveau complex of Zariski sheaves $\mathcal{C}(F)$ defined as

$$
\bigoplus_{x \in X_{1}^{(0)}} i_{x, *} H_{x}^{0}\left(X_{1}, \mathcal{F}\right) \rightarrow \bigoplus_{x \in X_{1}^{(1)}} i_{x, *} H_{x}^{1}\left(X_{1}, \mathcal{F}\right) \rightarrow \bigoplus_{x \in X_{1}^{(2)}} i_{x, *} H_{x}^{2}\left(X_{1}, \mathcal{F}\right) \rightarrow \cdots
$$

where the left group is put into cohomological degree 0 and where $i_{x}: x \rightarrow X$ is the natural monomorphism. There is a canonical augmentation $\mathcal{F} \rightarrow \mathcal{C}^{0}(\mathcal{F})$.

By $\mathrm{C}(\mathcal{F})$ we denote the complex of global sections $\Gamma(X, \mathcal{C}(\mathcal{F}))$.

Definition 3.2. An abelian sheaf $\mathcal{F}$ on $X_{1}$ is Cohen-Macaulay (CM for short) if for every scheme point $x \in X_{1}$ we have $H_{x}^{i}\left(X_{1}, \mathcal{F}\right)=0$ for $i \neq \operatorname{codim}(x)$.

A basic observation about CM-sheaves is that they give rise to exact coniveau complexes. This follows directly from the degeneration of the coniveau spectral sequence for these sheaves, see [Har66, Prop. IV.2.6].

Proposition 3.3. Let $\mathcal{F}$ be a $C M$ abelian Zariski sheaf. Then $\mathcal{C}(\mathcal{F})$ is an acyclic resolution of $\mathcal{F}$. In particular one has $H^{*}(X, \mathcal{F}) \cong H^{*}(\mathrm{C}(\mathcal{F}))$.

The aim of this section is to show the following result.

TheOrem 3.4. The sheaves $\mathcal{K}_{r, X_{n}}^{\mathrm{M}}, \Omega_{X_{n}}^{i}, \mathcal{H}_{X_{1}}^{r}, Z_{X_{n}}^{r}, B_{X_{n}}^{r}$ are $C M$ for all $n \geqslant 1$.

We prove the theorem in a series of propositions.

Proposition 3.5. Let $r, n \geqslant 0$ be integers. The sheaves $\mathcal{F}=\mathcal{K}_{r, X_{n}}^{\mathrm{M}}$ and $\mathcal{F}=\Omega_{X_{n}}^{r}$ are $C M$.

Proof. For the sheaf $\mathcal{F}=\Omega_{X_{1}}^{r}$ see [Har66, p. 239]. For the sheaf $\mathcal{F}=\mathcal{K}_{r, X_{1}}^{\mathrm{M}}$ see [Ker09]. We prove that $\mathcal{F}=\mathcal{K}_{r, X_{n}}^{\mathrm{M}}$ is $\mathrm{CM}$ by induction on $n$. The case of the sheaf $\mathcal{F}=\Omega_{X_{n}}^{r}$ works similarly. For $n>1$ we get from the exact sequence (2.8) an exact sequence

$$
H_{x}^{i}\left(X_{1}, \Omega_{X_{1}}^{r}\right) \rightarrow H_{x}^{i}\left(X_{1}, \mathcal{K}_{r, X_{n}}^{\mathrm{M}}\right) \rightarrow H_{x}^{i}\left(X_{1}, \mathcal{K}_{r, X_{n-1}}^{\mathrm{M}}\right) .
$$

For $i \neq \operatorname{codim}(x)$ we already know that the groups on the left and the right sides vanish, so does the group in the middle. 


\section{Deformation of CYCle Classes}

Next we study the sheaf $Z_{X_{1}}^{r}$.

Proposition 3.6. For any $r \geqslant 0$ and $x \in X_{1}$ the map

$$
H_{x}^{c}\left(X_{1}, B_{X_{1}}^{r}\right) \rightarrow H_{x}^{c}\left(X_{1}, Z_{X_{1}}^{r}\right)
$$

induced by the inclusion $B_{X_{1}}^{r} \subset Z_{X_{1}}^{r}$ is surjective for $c \neq \operatorname{codim}(x)$ and injective for $c \neq$ $\operatorname{codim}(x)+1$.

Proof. The short exact sequence of sheaves

$$
0 \rightarrow B_{X_{1}}^{r} \rightarrow Z_{X_{1}}^{r} \rightarrow, \mathcal{H}_{X_{1}}^{r} \rightarrow 0
$$

induces a long exact cohomology sequence

$$
\cdots \rightarrow H_{x}^{c-1}\left(X_{1}, \mathcal{H}_{X_{1}}^{r}\right) \rightarrow H_{x}^{c}\left(X_{1}, B_{X_{1}}^{r}\right) \rightarrow H_{x}^{c}\left(X_{1}, Z_{X_{1}}^{r}\right) \rightarrow H_{x}^{c}\left(X_{1}, \mathcal{H}_{X_{1}}^{r}\right) \rightarrow \cdots
$$

On the other hand, we known from [BO75] that the sheaves $\mathcal{H}_{X_{1}}^{r}$ are CM. This shows the proposition.

Proposition 3.7. The sheaves $B_{X_{1}}^{*}, Z_{X_{1}}^{*}$ are $C M$.

Proof. Using the exact sequence

$$
0 \rightarrow Z_{X_{1}}^{r} \rightarrow \Omega_{X_{1}}^{r} \rightarrow B_{X_{1}}^{r+1} \rightarrow 0
$$

and Proposition 3.5, we get

$$
H_{x}^{c}\left(X_{1}, Z_{X_{1}}^{r}\right)=H_{x}^{c-1}\left(X_{1}, B_{X_{1}}^{r+1}\right) \quad \text { for } c \notin\{\operatorname{codim}(x), \operatorname{codim}(x)+1\} .
$$

Combining Proposition 3.6 and (3.2) we get for $c>\operatorname{codim}(x)$ the identifications

$$
\begin{aligned}
H_{x}^{c}\left(X_{1}, Z_{X_{1}}^{r}\right) & \leftarrow H_{x}^{c}\left(X_{1}, B_{X_{1}}^{r}\right)=H_{x}^{c+1}\left(X_{1}, Z_{X_{1}}^{r-1}\right) \\
& =H_{x}^{c+1}\left(X_{1}, B_{X_{1}}^{r-1}\right)=H_{x}^{c+2}\left(X_{1}, Z_{X_{1}}^{r-2}\right)=\cdots=0,
\end{aligned}
$$

where the first arrow surjective. For $c<\operatorname{codim}(x)$, we get the identifications

$$
\begin{aligned}
H_{x}^{c}\left(X_{1}, B_{X_{1}}^{r}\right) & =H_{x}^{c}\left(X_{1}, Z_{X_{1}}^{r}\right)=H_{x}^{c-1}\left(X_{1}, B_{X_{1}}^{r+1}\right)=H_{x}^{c-1}\left(X_{1}, Z_{X_{1}}^{r+1}\right) \\
& =H_{x}^{c-2}\left(X_{1}, B_{X_{1}}^{r+2}\right)=H_{x}^{c-2}\left(X_{1}, Z_{X_{1}}^{r+2}\right)=\cdots=0 .
\end{aligned}
$$

Corollary 3.8. For all $n \geqslant 1$, the sheaves $B_{X_{n}}^{*}, Z_{X_{n}}^{*}$ are $C M$.

Proof. The sheaf $Z_{X_{n}}^{*}$ is CM by Propositions 2.3, 3.5 and 3.7. Using Lemma 2.4 and the fact that $B_{X_{n}}^{r}$ trivially surjects onto $B_{X_{n-1}}^{r}$, we get a commutative diagram of short exact sequences

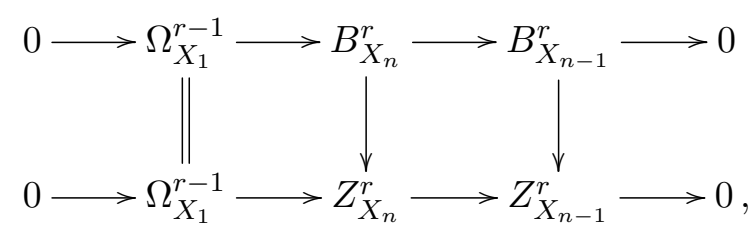

which implies that $B_{X_{n}}^{*}$ is CM.

\section{Transfer map}

For any algebraic scheme $V$, it is natural to define the (cohomological) Chow groups

$$
\mathrm{CH}^{p}(V):=H^{p}\left(V, \mathcal{K}_{p}^{\mathrm{M}}\right) \text {. }
$$




\section{Bloch, Esnault and Kerz}

In this way, the graded object $\mathrm{CH}^{*}(V)$ is automatically a ring, contravariant in $V$. For regular excellent $V$ the cohomology group $\mathrm{CH}^{i}(V)$ coincides with the usual Chow group of codimension $i$ cycles on $V$ by [Ker09]. We would hope there exist covariant transfer maps $\mathrm{CH}^{*}(V) \stackrel{f_{*}}{\rightarrow} \mathrm{CH}^{*}(W)$ of graded degree $-d$ for $f: V \rightarrow W$ smooth and proper with fiber dimension $d$. One might further hope for $f$ proper and $d=\operatorname{dim} V-\operatorname{dim} W$ that there exists a functorial map of coniveau complexes $\mathrm{C}\left(\mathcal{K}_{V, r}^{\mathrm{M}}\right) \stackrel{f_{*}}{\rightarrow} \mathrm{C}\left(\mathcal{K}_{W, r-d}^{\mathrm{M}}\right)[-d]$ such that when $\mathcal{K}_{W, r-d}^{\mathrm{M}}$ is $\mathrm{CM}$ one could define a covariant transfer via

$$
H^{p}\left(V, \mathcal{K}_{p}^{\mathrm{M}}\right) \rightarrow H^{p}\left(\mathrm{C}\left(\mathcal{K}_{p, V}^{\mathrm{M}}\right)\right) \stackrel{f_{*}}{\rightarrow} H^{p-d}\left(\mathrm{C}\left(\mathcal{K}_{W, p-d}^{\mathrm{M}}\right)\right) \cong H^{p-d}\left(W, \mathcal{K}_{W, p-d}^{\mathrm{M}}\right) .
$$

In what follows we use results from the previous section, together with work of Rost [Ros96] and Grothendieck [Har66], to define a transfer

$$
f_{*}: \mathrm{CH}^{i}\left(X_{n}\right) \rightarrow \mathrm{CH}^{i-d}\left(Y_{n}\right)
$$

for $f: X_{n} \rightarrow Y_{n}$ a smooth proper morphism of relative dimension $d$ between smooth schemes over $S_{n}$. This suffices to define a calculus of correspondences on $\mathrm{CH}^{*}\left(X_{n}\right)$, which is what we will need.

We use the fiber square in Proposition 2.7 to 'glue' the constructions of Rost and Grothendieck. From Propositions 2.7 and 3.5 and Corollary 3.8 we obtain the following results.

Lemma 4.1. There is a Cartesian square of complexes

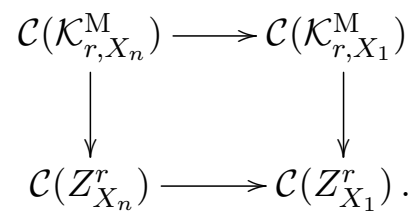

Lemma 4.2. We have a left-exact sequence

$$
0 \rightarrow \mathcal{C}\left(Z_{X_{n}}^{r}\right) \rightarrow \mathcal{C}\left(\Omega_{X_{n}}^{r}\right) \stackrel{d}{\rightarrow} \mathcal{C}\left(\Omega_{X_{n}}^{r+1}\right) .
$$

Proof. A short exact sequence of $C M$ sheaves yields a short exact sequence of coniveau complexes. Applying this to the sequence

$$
0 \rightarrow Z_{X_{n}}^{r} \rightarrow \Omega_{X_{n}}^{r} \rightarrow B_{X_{n}}^{r+1} \rightarrow 0
$$

we reduce the problem to showing that the map $\mathcal{C}\left(B_{X_{n}}^{r+1}\right) \rightarrow \mathcal{C}\left(\Omega_{X_{n}}^{r+1}\right)$ is injective. By the same logic, we know that $\mathcal{C}\left(Z_{X_{n}}^{r+1}\right) \hookrightarrow \mathcal{C}\left(\Omega_{X_{n}}^{r+1}\right)$, so it suffices to show that $\mathcal{C}\left(B_{X_{n}}^{r+1}\right) \hookrightarrow \mathcal{C}\left(Z_{X_{n}}^{r+1}\right)$. By Lemma 2.4 we have an exact sequence

$$
0 \rightarrow B_{X_{n}}^{r+1} \rightarrow Z_{X_{n}}^{r+1} \rightarrow \mathcal{H}_{X_{1}}^{r+1} \rightarrow 0
$$

where $\mathcal{H}_{X_{1}}^{r+1}$ is CM. We conclude that $\mathcal{C}\left(B_{X_{n}}^{r+1}\right) \hookrightarrow \mathcal{C}\left(Z_{X_{n}}^{r+1}\right)$, proving the lemma.

Let now $f: X_{n} \rightarrow Y_{n}$ be, as above, a smooth proper map of relative dimension $d$ between smooth schemes over $S_{n}$. Rost constructs a morphism between complexes [Ros96] which, via the Gysin isomorphisms, is a transfer

$$
f_{*} \mathcal{C}\left(\mathcal{K}_{r, X_{1}}^{\mathrm{M}}\right) \stackrel{f_{*}}{\rightarrow} \mathcal{C}\left(\mathcal{K}_{r-d, Y_{1}}^{\mathrm{M}}\right)[-d] .
$$

Grothendieck and Hartshorne construct in [Har66] a morphism between complexes of $\mathcal{O}_{Y_{n}}{ }^{-}$ modules

$$
f_{*} \mathcal{C}\left(\Omega_{X_{n} / Y_{n}}^{d}\right) \stackrel{\operatorname{Tr}_{f}}{\longrightarrow} \mathcal{C}\left(\mathcal{O}_{Y_{n}}\right)[-d]
$$




\section{Deformation of CYCle Classes}

Indeed, using the notation of [Har66], the structure sheaf $\mathcal{O}_{Y_{n}}$ is pointwise dualizing, so its coniveau complex $\mathcal{C}\left(\mathcal{O}_{Y_{n}}\right)$ is a residual complex and

$$
f^{!} \mathcal{C}\left(\mathcal{O}_{Y_{n}}\right)=\mathcal{C}\left(\Omega_{X_{n} / Y_{n}}^{d}\right)[d]
$$

Now we consider the composite morphism

$$
f_{*}: f_{*} \mathcal{C}\left(\Omega_{X_{n}}^{r}\right) \rightarrow \Omega_{Y_{n}}^{r-d} \otimes_{\mathcal{O}_{Y_{n}}} f_{*} \mathcal{C}\left(\Omega_{X_{n} / Y_{n}}^{d} \stackrel{\operatorname{Tr}_{f}}{\longrightarrow} \Omega_{Y_{n}}^{r-d} \otimes_{\mathcal{O}_{Y_{n}}} \mathcal{C}\left(\mathcal{O}_{Y_{n}}\right)[-d] \stackrel{\sim}{\rightarrow} \mathcal{C}\left(\Omega_{Y_{n}}^{r-d}\right)[-d]\right.
$$

where for the first arrow we use the projection $\Omega_{X_{n}}^{r} \rightarrow f^{*} \Omega_{Y_{n}}^{r-d} \otimes_{\mathcal{O}_{X_{n}}} \Omega_{X_{n} / Y_{n}}^{d}$ followed by the projection formula. Note that the composite map $f_{*}$ in (4.4) is compatible with the differential.

One directly shows that the transfer map (4.4) for $n=1$ is compatible with the transfer map (4.2) with respect to the $d \log$ map. The transfer (4.4) induces thanks to Lemma 4.2 a transfer

$$
\mathrm{C}\left(Z_{X_{n}}^{r}\right) \stackrel{f_{*}}{\rightarrow} \mathrm{C}\left(Z_{Y_{n}}^{r-d}\right)[-d]
$$

So we get a commutative diagram of exact sequences

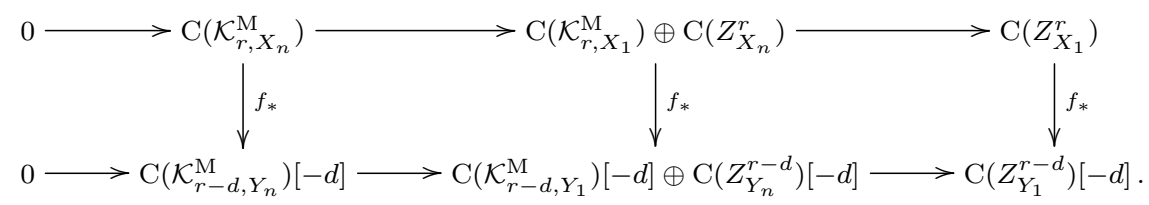

The left vertical transfer map is defined by this diagram. The transfer map

$$
f_{*}: \mathrm{CH}^{i}\left(X_{n}\right) \cong H^{i}\left(\mathrm{C}\left(\mathcal{K}_{i, X_{n}}^{\mathrm{M}}\right)\right) \rightarrow H^{i-d}\left(\mathrm{C}\left(\mathcal{K}_{i-d, Y_{n}}^{\mathrm{M}}\right)\right) \cong \mathrm{CH}^{i-d}\left(Y_{n}\right),
$$

obtained using the above construction, Proposition 3.3 and Theorem 3.4, satisfies the usual properties, for example it is compatible with smooth base change, and we use such properties without further mentioning.

For the remaining part of this section let $d$ be the dimension of the smooth, equidimensional scheme $X_{n} / S_{n}$. If one follows the above construction of the transfer carefully, one can deduce the following result.

\section{PROPOSITION 4.3.}

(i) Composition of correspondences makes $\mathrm{CH}^{d}\left(X_{n} \times_{S_{n}} X_{n}\right)$ into a ring with unity for any $n \geqslant 1$.

(ii) For $n \geqslant 2$ this ring acts canonically on the long exact cohomology sequence

$$
\cdots \rightarrow H^{c}\left(X_{1}, \Omega_{X_{1}}^{r-1}\right) \rightarrow H^{c}\left(X_{n} ; \mathcal{K}_{r}^{\mathrm{M}}\right) \rightarrow H^{c}\left(X_{n-1} ; \mathcal{K}_{r}^{\mathrm{M}}\right) \rightarrow H^{c+1}\left(X_{1}, \Omega_{X_{1}}^{r-1}\right) \rightarrow \cdots
$$

associated with (2.8) for any integer $r \geqslant 0$.

(iii) The kernel $\operatorname{ker}\left[\mathrm{CH}^{d}\left(X_{n} \times_{S_{n}} X_{n}\right) \rightarrow \mathrm{CH}^{d}\left(X_{1} \times_{k} X_{1}\right)\right]$ is a nilpotent ideal.

Proposition 4.4. Fix a positive integer $i$ and assume condition $(\mathbf{C K})_{X_{\eta}}^{2 i}$ from the introduction. Then there exists an inverse system of correspondences $\left(\pi_{n}^{2 i}\right)_{n \geqslant 1}$ with $\pi_{n}^{2 i} \in \mathrm{CH}^{d}\left(X_{n} \times_{S_{n}} X_{n}\right)_{\mathbb{Q}}$ such that the following properties hold:

(i) each $\pi_{n}^{2 i}$ is idempotent;

(ii) on $H_{\mathrm{dR}}^{*}\left(X_{n} / S_{n}\right)$ the correspondence $\pi_{n}^{2 i}$ acts as the projection to $H^{2 i}\left(X_{n} / S_{n}\right)$.

Proof. Consider $\pi_{\eta}^{2 i} \in \mathrm{CH}^{d}\left(X_{\eta} \times_{K} X_{\eta}\right)_{\mathbb{Q}}$ as in property $(\mathbf{C K})_{X_{\eta}}^{2 i}$. The element $\pi_{1}^{2 i}$ is defined as the specialization of $\pi_{\eta}^{2 i}$ to the reduced closed fiber $X_{1}$. Recall that the specialization map

$$
\mathrm{CH}^{d}\left(X_{\eta} \times_{K} X_{\eta}\right) \rightarrow \mathrm{CH}^{d}\left(X_{1} \times_{k} X_{1}\right)
$$




\section{Bloch, Esnault and Kerz}

is a ring homomorphism of correspondences [Ful84, Sec. 20.3].

As a consequence, $\pi_{1}^{2 i}$ satisfies properties (i) and (ii) of Proposition 4.4 for $n=1$. We claim that we can lift this element to an inverse system $\left(\pi_{n}^{2 i}\right)_{n \geqslant 1}$ with the requested properties.

We can extend $\pi_{\eta}^{2 i}$ to an element $\pi^{2 i} \in \mathrm{CH}^{d}\left(X \times_{S} X\right)_{\mathbb{Q}}$. By the Gersten conjecture for the Milnor $K$-sheaf of the regular scheme $X \times{ }_{S} X$ [Ker09] we get the left isomorphism in the following diagram

$$
\begin{aligned}
\iota_{n}: \mathrm{CH}^{d}\left(X \times_{S} X\right) & \cong H^{d}\left(X \times_{S} X, \mathcal{K}_{d, X \times{ }_{S} X}^{\mathrm{M}}\right) \longrightarrow \\
& H^{d}\left(X_{n} \times{ }_{S_{n}} X_{n}, \mathcal{K}_{d, X_{n} \times{ }_{S_{n}} X_{n}}^{\mathrm{M}}\right)=\mathrm{CH}^{d}\left(X_{n} \times_{S_{n}} X_{n}\right) .
\end{aligned}
$$

Now consider the inverse system of correspondences $\tilde{\pi}_{n}^{2 i}=\iota_{n}\left(\pi^{2 i}\right)$. They satisfy property (ii) of the proposition and furthermore $\pi_{1}^{2 i}=\tilde{\pi}_{1}^{2 i}$. We will apply a transformation to these correspondences which additionally makes them idempotent.

For an element $\alpha$ of an arbitrary (not necessarily commutative) unital ring and an integer $s \geqslant 1$ set

$$
f_{s}(\alpha)=\sum_{0 \leqslant j \leqslant s}\left(\begin{array}{c}
2 s \\
j
\end{array}\right) \alpha^{2 s-j}(1-\alpha)^{j} .
$$

From the argument in the proof of [Bas68, Prop. III.2.10] and from Proposition 4.3(iii) it follows that for $s$ large, depending on $n$, the element $\pi_{n}^{2 i}=f_{s}\left(\tilde{\pi}_{n}^{2 i}\right)$ is idempotent and independent of $s \gg 0$. Observe that $f_{s}\left(\pi_{1}^{2 i}\right)=\pi_{1}^{2 i}$ for all $s \geqslant 1$, because $\pi_{1}^{2 i}$ is idempotent. The elements $\left(\pi_{n}^{2 i}\right)_{n \geqslant 1}$ form an inverse system of idempotent correspondences, finishing the proof of Proposition 4.4.

In the next section we use the action of a Künneth type correspondence on the cohomology of absolute differential forms as described in the following proposition.

Proposition 4.5. Consider for given $i \geqslant 0$ a correspondence $\pi^{i} \in \mathrm{CH}^{d}\left(X_{1} \times_{k} X_{1}\right)_{\mathbb{Q}}$ which acts on $H_{\mathrm{dR}}^{*}\left(X_{1} / k\right)$ as the projection to $H_{\mathrm{dR}}^{i}\left(X_{1} / k\right)$. Then

(i) the action of the correspondence $\left(\pi^{i}\right)^{r+1}$ on $H^{c}\left(X_{1}, \Omega_{X_{1}}^{r}\right)$ vanishes for all $c+r<i$;

(ii) the action of $\left(\pi^{i}\right)^{r}$ on

$$
\operatorname{ker}\left[H^{c}\left(X_{1}, \Omega_{X_{1}}^{r}\right) \rightarrow H^{c}\left(X_{1}, \Omega_{X_{1} / k}^{r}\right)\right]
$$

vanishes for $c+r=i$.

Proof. The correspondence $\pi^{i}$, being algebraic, respects the Hodge filtration on de Rham cohomology and therefore acts on its graded pieces, which are Hodge cohomology groups according to Hodge theory. Thus $\pi^{i}$ acts trivially on $H^{c}\left(X_{1}, \Omega_{X_{1} / k}^{r}\right)$ for $c+r<i$. To pass from relative differential forms to absolute differential forms we use the filtration

$$
L^{s} \Omega_{X_{1}}^{r}=\operatorname{im}\left[\Omega_{k}^{s} \otimes_{k} \Omega_{X_{1}}^{r-s} \rightarrow \Omega_{X_{1}}^{r}\right] \quad(s=0, \ldots, r) .
$$

Recall that

$$
L^{s} / L^{s+1} \Omega_{X_{1}}^{r}=\Omega_{X_{1} / k}^{r-s} \otimes_{k} \Omega_{k}^{s}
$$

and that $\pi^{i}$ acts on the system of morphisms

$$
H^{c}\left(X_{1}, \Omega_{k}^{r} \otimes_{k} \mathcal{O}_{X_{1}}\right) \rightarrow H^{c}\left(X_{1}, L^{r-1} \Omega_{X_{1}}^{r}\right) \rightarrow H^{c}\left(X_{1}, L^{r-2} \Omega_{X_{1}}^{r}\right) \rightarrow \cdots \rightarrow H^{c}\left(X_{1}, \Omega_{X_{1}}^{r}\right) .
$$

The filtration on cohomology

$$
L^{s}=\operatorname{im}\left[H^{c}\left(X_{1}, L^{s} \Omega_{X_{1}}^{r}\right) \rightarrow H^{c}\left(X_{1}, \Omega_{X_{1}}^{r}\right)\right]
$$




\section{Deformation of CYCle ClASSES}

has graded pieces $L^{s} / L^{s+1}$ which are subquotients of

$$
H^{c}\left(X_{1}, \Omega_{X_{1} / k}^{r-s}\right) \otimes_{k} \Omega_{k}^{s} .
$$

On the latter groups the action of $\pi^{i}$ vanishes for $c+r-s<i$ as we have seen above.

This shows property (i), while property (ii) follows from the additional observation that

$$
L^{1}=\operatorname{ker}\left[H^{c}\left(X_{1}, \Omega_{X_{1}}^{r}\right) \rightarrow H^{c}\left(X_{1}, \Omega_{X_{1} / k}^{r}\right)\right] .
$$

\section{Deformation of Chow groups}

Let the notation be as in Section 2. We start this section with a basic lemma from [Blo72] about the comparison of an obstruction map with a Kodaira-Spencer map.

LEMMA 5.1. The diagram

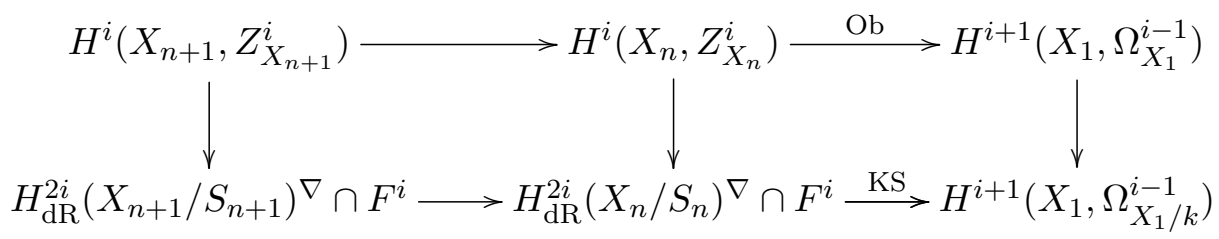

is commutative with exact rows.

Here $\nabla \in \operatorname{End}_{k}\left(H_{\mathrm{dR}}^{*}\left(X_{n} / S_{n}\right)\right)$ is the Gauss-Manin connection and $F^{i}$ is the Hodge filtration. The upper row is part of the long exact cohomology sequence associated with (2.8) and KS is induced by the Kodaira-Spencer map [Blo72, (4.1)].

We can now state the version of our main theorem for (cohomological) Chow groups. Let

$$
\mathrm{cl}: \mathrm{CH}^{i}\left(X_{n}\right) \rightarrow H_{\mathrm{dR}}^{2 i}\left(X_{n} / S_{n}\right)^{\nabla} \subset H_{\mathrm{dR}}^{2 i}\left(X_{n} / S_{n}\right)
$$

be the de Rham cycle class map, which is induced by the morphism of complexes

$$
d \log : \mathcal{K}_{i}^{\mathrm{M}}[-i] \rightarrow \Omega_{X_{n} / S_{n}} .
$$

The restriction map

$$
\Phi: H_{\mathrm{dR}}^{2 i}(X / S)^{\nabla \stackrel{\sim}{\rightarrow}} H_{\mathrm{dR}}^{2 i}\left(X_{1} / k\right)
$$

is an isomorphism by [Kat70, Prop. 8.9].

Theorem 5.2. Assume that for $X / S$ as above and for a fixed $i$ the property $(\mathbf{C K})_{X_{\eta}}^{2 i}$ explained in the introduction holds. Then for $\xi_{1} \in \mathrm{CH}^{i}\left(X_{1}\right)_{\mathbb{Q}}$ the following are equivalent:

(i) $\Phi^{-1} \circ \operatorname{cl}\left(\xi_{1}\right) \in H_{\mathrm{dR}}^{2 i}(X / S)^{\nabla} \cap F^{i} H_{\mathrm{dR}}^{2 i}(X / S)$;

(ii) there is an element $\hat{\xi} \in\left(\lim _{n} \mathrm{CH}^{i}\left(X_{n}\right)\right)_{\mathbb{Q}}$ such that

$$
\operatorname{cl}\left(\left.\hat{\xi}\right|_{X_{1}}\right)=\operatorname{cl}\left(\xi_{1}\right) \in H_{\mathrm{dR}}^{2 i}\left(X_{1} / k\right) .
$$

Remark 5.3. For $k$ algebraic over $\mathbb{Q}$ it has been known to the experts for a long time (see [GG04], and [PR13] for more recent work in the case of hypersurface sections) that for an element $\xi_{1} \in \mathrm{CH}^{i}\left(X_{1}\right)$ (note that we can use integral coefficients here) condition (i) of the theorem is equivalent to:

(ii') there is an element $\hat{\xi} \in \lim _{\longleftarrow} \mathrm{CH}^{i}\left(X_{n}\right)$ such that $\left.\xi\right|_{X_{1}}=\xi_{1}$. 


\section{Bloch, Esnault and Kerz}

Proof of Theorem 5.2. The implication condition (ii) $\Rightarrow$ condition (i) is clear. So consider condition (i) $\Rightarrow$ condition (ii).

Claim 5.4. The map

$$
(\underbrace{\lim }_{n} \mathrm{CH}^{i}\left(X_{n}\right))_{\mathbb{Q}} \rightarrow \underset{n}{\lim _{n}} \mathrm{CH}^{i}\left(X_{n}\right)_{\mathbb{Q}}
$$

is surjective.

Proof. From the short exact sequence (2.8) we get for $n>1$ a commutative diagram with exact sequences

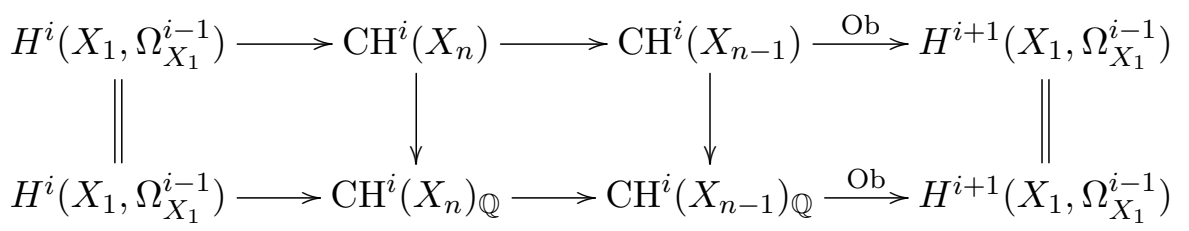

A diagram chase implies that the map

$$
\mathrm{CH}^{i}\left(X_{n}\right) \longrightarrow \mathrm{CH}^{i}\left(X_{n-1}\right) \times{ }_{\mathrm{CH}^{i}\left(X_{n-1}\right)_{\mathbb{Q}}} \mathrm{CH}^{i}\left(X_{n}\right)_{\mathbb{Q}}
$$

is surjective. From this Claim 5.4 follows easily.

By Claim 5.4 it is enough to construct a pro-system $\hat{\xi} \in \lim _{n} \mathrm{CH}^{i}\left(X_{n}\right)_{\mathbb{Q}}$ satisfying (5.1). We will do this successively.

Choose correspondences $\pi_{n}^{2 i}$ as in Proposition 4.4. We claim that there exists an element

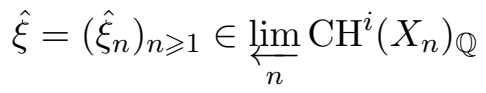

such that

$$
\hat{\xi}_{1}=\pi_{1}^{2 i} \cdot \xi_{1} \quad \text { and } \quad \pi_{n}^{2 i} \cdot \hat{\xi}_{n}=\hat{\xi}_{n} \quad \text { for all } n \geqslant 1 .
$$

Indeed, assume that we have already constructed $\left(\hat{\xi}_{m}\right)_{1 \leqslant m \leqslant n-1}$ with property (5.3). From Proposition 4.3 we know that $\pi_{n}^{2 i}$ acts on the following diagram with exact row and column

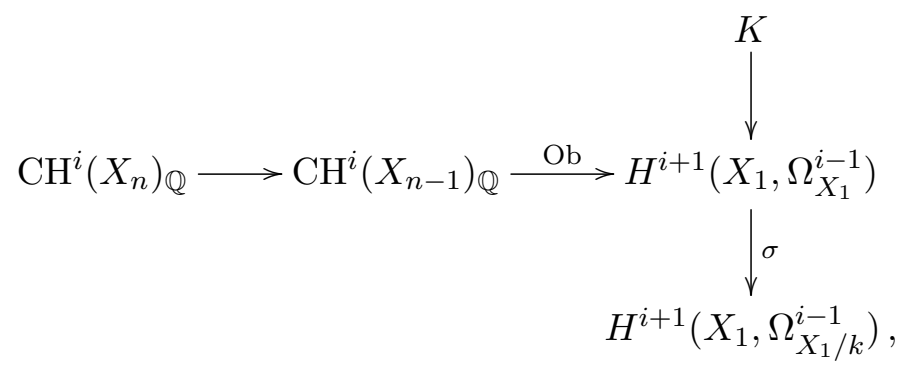

where $K$ is defined as the kernel of $\sigma$. By Lemma 5.1 we have $\operatorname{Ob}\left(\hat{\xi}_{n-1}\right) \in K$. From the latter and Proposition 4.5 we deduce the third equality in

$$
\operatorname{Ob}\left(\hat{\xi}_{n-1}\right)=\operatorname{Ob}\left(\left(\pi_{n-1}^{2 i}\right)^{i-1} \cdot \hat{\xi}_{n-1}\right)=\left(\pi_{1}^{2 i}\right)^{i-1} \cdot \operatorname{Ob}\left(\hat{\xi}_{n-1}\right)=0 .
$$

Because the obstruction vanishes we can find $\hat{\xi}_{n}^{\prime} \in \mathrm{CH}^{i}\left(X_{n}\right)_{\mathbb{Q}}$ with $\left.\hat{\xi}_{n}^{\prime}\right|_{X_{n-1}}=\hat{\xi}_{n-1}$. To finish the construction we set $\hat{\xi}_{n}=\pi_{n}^{2 i} \cdot \hat{\xi}_{n}^{\prime}$. 


\section{DEFORMATION OF CYCle ClASSES}

\section{Motivic complex and Chern character}

We begin this section by proving that the canonical map from Milnor $K$-theory to Quillen $K$ theory induces an isomorphism on certain relative $K$-groups. Then we study a Chern character isomorphism using higher algebraic $K$-theory and motivic cohomology. For both results the techniques are standard, so we only sketch the proofs.

We consider a pro-system of pairs of rings of the form $\left(A_{\bullet}, A\right)$ with $A_{\bullet}=A[t] /\left(t^{n}\right)$ and we assume that $A$ is essentially smooth over $k$ with $\operatorname{char}(k)=0$. By $K_{*}\left(R^{\prime}, R\right)$ we denote the relative Quillen $K$-groups of a homomorphism between rings $R^{\prime} \rightarrow R$.

Proposition 6.1. For $A$ as above the canonical homomorphism

$$
\operatorname{ker}\left[K_{*}^{\mathrm{M}}\left(A_{\bullet}\right) \rightarrow K_{*}^{\mathrm{M}}(A)\right] \stackrel{\sim}{\rightarrow} K_{*}\left(A_{\bullet}, A\right)
$$

is a pro-isomorphism.

Proof. By Goodwillie's theorem [Goo86] there is an isomorphism

$$
K_{i+1}\left(A_{n}, A\right) \stackrel{\sim}{\rightarrow} \mathrm{HC}_{i}\left(A_{n}, A\right)
$$

for any $n \geqslant 1$. There is a canonical homomorphism

$$
e_{n}: \mathrm{HC}_{i}\left(A_{n}\right) \rightarrow \Omega_{A_{n}}^{i} / B_{A_{n}}^{i} \oplus Z_{A_{n}}^{i-2} / B_{A_{n}}^{i-2} \oplus Z_{A_{n}}^{i-4} / B_{A_{n}}^{i-4} \oplus \cdots,
$$

see [Wei94, 9.8]. By the Hochschild-Kostant-Rosenberg theorem [Wei94, Thm. 9.4.7] and a proversion of it (see [Mor13b] for a general discussion), one sees that the corresponding maps on Hochschild homology

$$
e: \mathrm{HH}_{*}(A) \rightarrow \Omega_{A}^{*} \quad \text { and } \quad e: \mathrm{HH}_{*}\left(A_{\bullet}\right) \rightarrow \Omega_{A}^{*}
$$

induce an isomorphism and a pro-isomorphism, respectively. Then by a short argument with mixed complexes [Wei94, 9.8.13] one deduces that the map $e_{1}$ and the pro-system of maps $e_{\bullet}$ from (6.3) induce an isomorphism and a pro-isomorphism, respectively. Finally, using Lemma 2.4 we see that we get pro-isomorphisms

$$
\mathrm{HC}_{i}\left(A_{\bullet}, A\right) \stackrel{e_{\bullet}}{\rightarrow} \operatorname{ker}\left[\Omega_{A \bullet}^{i} / B_{A \bullet}^{i} \rightarrow \Omega_{A}^{i} / B_{A}^{i}\right] \stackrel{d}{\rightarrow} \operatorname{ker}\left[Z_{A_{\bullet}}^{i+1} \rightarrow Z_{A}^{i+1}\right] .
$$

Following the steps of this construction carefully shows that the composition of (6.1), (6.2) and (6.4) is equal to the $d \log$ map, which is an isomorphism by the Cartesian square (2.7).

More general results in the direction of Proposition 6.1 can be found in [Mor13a] and [Mor13b].

By classical techniques one constructs a Chern character ring homomorphism

$$
\operatorname{ch}: K_{0}\left(X_{n}\right)_{\mathbb{Q}} \rightarrow \bigoplus_{i \geqslant 0} \mathrm{CH}^{i}\left(X_{n}\right)_{\mathbb{Q}},
$$

where we use the notation of Section 2. The Chern character is characterized by the property that the composite morphism

$$
H^{1}\left(X_{1}, \mathcal{O}_{X_{n}}^{\times}\right) \cong \operatorname{Pic}\left(X_{n}\right) \rightarrow K_{0}\left(X_{n}\right)_{\mathbb{Q}} \stackrel{\mathrm{ch}}{\rightarrow} \bigoplus_{i \geqslant 0} \mathrm{CH}^{i}\left(X_{n}\right)_{\mathbb{Q}} \rightarrow \mathrm{CH}^{1}\left(X_{n}\right)_{\mathbb{Q}}
$$

is induced by the canonical isomorphisms $H^{1}\left(X_{1}, \mathcal{O}_{X_{n}}^{\times}\right) \cong \mathrm{CH}^{1}\left(X_{n}\right)$.

Using Proposition 6.1 and Zariski descent for algebraic $K$-theory [TT90, Sec. 10] we will show in this section that (6.5) induces a pro-isomorphism with respect to $n$, see Theorem 6.2. The pro-isomorphism (6.5) together with Theorem 5.2 immediately imply Theorem 1.2. 


\section{Bloch, Esnault and Kerz}

Let $\mathbb{Z}_{X_{1}}(r)$ be the weight $r$ motivic complex of Zariski sheaves on the smooth variety $X_{1} / k$ constructed by Suslin-Voevodsky, see [MVW06]. We define a motivic complex $\mathbb{Z}_{X_{n}}(r)$ of the scheme $X_{n}$ by the homotopy Cartesian square

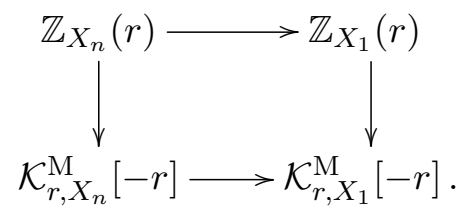

The reader should be warned that the complex $\mathbb{Z}_{X_{n}}(r)$ for fixed $n$ cannot be 'the correct' motivic complex of $X_{n}$, but as a pro-system in $n$ we get the 'right' motivic theory. In fact from comparison with algebraic $K$-theory we expect the 'proper' homotopy fiber of the upper row of (6.6) to have nontrivial cohomology sheaves in each degree in the interval $[1, r]$ and not only in degree $r$.

There is a Chern character homomorphism from (higher) algebraic $K$-theory to the cohomology of the motivic complex $\mathbb{Z}_{X \bullet}(r)$. The technique of the construction of the higher Chern character is explained in [Gil81]. We recall the construction.

The universal Chern character

$$
\operatorname{ch} \in \bigoplus_{r \geqslant 0} H^{2 r}\left(\mathrm{BGL}_{k}, \mathbb{Q}_{X_{1}}(r)\right)
$$

induces morphisms

$$
\operatorname{ch}_{r}: K_{X \bullet} \rightarrow \mathfrak{K Q}_{X}(r)[2 r]
$$

in the homotopy category of pro-spectra in the sense of [Isa04]. Here $K\left(X_{n}\right)$ is the nonconnective $K$-theory spectrum of $X_{n}$ [TT90, Sec. 6] and $\mathfrak{K}$ is the Eilenberg-MacLane functor.

Moreover, ch induces morphisms of Zariski descent spectral sequences [TT90, Sec. 10]

$$
\begin{aligned}
& { }^{K} E_{2}^{i, j}\left(X_{\bullet}\right)=H^{i}\left(X_{1}, \mathcal{K}_{-j, X \bullet}\right) \Rightarrow K_{-i-j, X \bullet} \\
& { }^{\mathrm{mot}} E_{2}^{i, j}\left(X_{\bullet}\right)=H^{i}\left(X_{1}, \mathcal{H}^{j}\left(\mathbb{Z}_{X \bullet}(r)\right)\right) \Rightarrow H^{i+j}\left(X_{1}, \mathbb{Z}_{X \bullet}(r)\right)
\end{aligned}
$$

of the form

$$
\operatorname{ch}_{r}:{ }^{K} E_{2}^{i, j}\left(X_{\bullet}\right)_{\mathbb{Q}} \rightarrow{ }^{\operatorname{mot}} E_{2}^{i, j+2 r}\left(X_{\bullet}\right)_{\mathbb{Q}}
$$

For any $r \geqslant 0$ there is a similar Chern character of relative theories

$$
\operatorname{ch}_{r}:{ }^{K} E_{2}^{i, j}\left(X_{\bullet}, X_{1}\right) \rightarrow{ }^{\operatorname{mot}} E_{2}^{i, j+2 r}\left(X_{\bullet}, X_{1}\right)
$$

which is a pro-isomorphism for $r=-j$ by Proposition 6.1 and vanishes otherwise. It is well known [Blo86] that the Chern character induces an isomorphism

$$
\operatorname{ch}: K_{i}\left(X_{1}\right)_{\mathbb{Q}} \stackrel{\sim}{\rightarrow} \bigoplus_{r \geqslant 0} H^{2 r-i}\left(X_{1}, \mathbb{Q}_{X_{1}}(r)\right)
$$

for any $i \in \mathbb{Z}$. Combining isomorphisms (6.9) and (6.10) we get the required isomorphism between pro-groups.

Theorem 6.2. For any smooth scheme $X / S$ which is separated and of finite type, there is a pro-isomorphism

$$
\operatorname{ch}: K_{i}\left(X_{\bullet}\right)_{\mathbb{Q}} \stackrel{\sim}{\rightarrow} \bigoplus_{r \geqslant 0} H^{2 r-i}\left(X_{1}, \mathbb{Q}_{X} \cdot(r)\right) .
$$

Observing that for any $n \geqslant 1$ there is a canonical isomorphism

$$
H^{2 r}\left(X_{1}, \mathbb{Z}_{X_{n}}(r)\right) \stackrel{\sim}{\rightarrow} \mathrm{CH}^{r}\left(X_{n}\right),
$$




\section{DEFORMATION OF CYCle ClASSES}

we see that the pro-isomorphism (6.11) comprises the pro-isomorphism (6.5).

\section{Appendix A. Two versions of Grothendieck's conjecture}

In the introduction we stated as Conjecture 1.4 a local version of Grothendieck's principle of the parallel transport of cycles, which we will refer to as the infinitesimal Hodge conjecture in the following. His original formulation, today called the variational Hodge conjecture, is more global and we show in this appendix that the two formulations are equivalent.

Let $k$ be a field of characteristic 0 . Let $f: \mathcal{X} \rightarrow \mathcal{S}$ be a smooth projective morphism, where $\mathcal{S} / k$ is a smooth variety. Fix a point $s \in \mathcal{S}$ and let $\mathcal{X}_{s}$ be the fiber over $s$.

Grothendieck's original conjecture [Gro66, p. 103] can now be stated as follows.

Conjecture A.1 (Variational Hodge). For $\xi_{s} \in K_{0}\left(\mathcal{X}_{s}\right)_{\mathbb{Q}}$ the following are equivalent:

(i) $\operatorname{ch}\left(\xi_{s}\right) \in H_{\mathrm{dR}}^{*}\left(\mathcal{X}_{s} / s\right)$ lifts to an element in $H_{\mathrm{dR}}^{*}(\mathcal{X} / k)$;

(ii) there is an element $\xi \in K_{0}(\mathcal{X})_{\mathbb{Q}}$ with $\operatorname{ch}\left(\left.\xi\right|_{\mathcal{X}_{s}}\right)=\operatorname{ch}\left(\xi_{s}\right)$.

Proposition A.2. The variational Hodge conjecture (Conjecture A.1) for all $k, \mathcal{X}, \mathcal{S}$ as above is equivalent to the infinitesimal Hodge conjecture (Conjecture 1.4) for all $k, X$ as in the introduction.

Remark A.3. The same proof shows that the variational Hodge conjecture for abelian schemes $\mathcal{X} / \mathcal{S}$ is equivalent to the infinitesimal Hodge conjecture for abelian schemes $X / S$.

Proof. Infinitesimal Hodge $\Longrightarrow$ Variational Hodge:

By induction on $\operatorname{dim}(\mathcal{S})$ we will reduce to $\operatorname{dim}(\mathcal{S})=1$. In order to do this observe first that we can replace without loss of generality $\mathcal{S}$ by a dense open subscheme containing $s$ and $\mathcal{X}$ by the corresponding pullback.

Now let $\xi_{s}$ satisfy Conjecture A.1(i) and assume without loss of generality that $\operatorname{codim}(s)>0$. Choose a smooth hypersurface $\mathcal{S}^{\prime} \subset \mathcal{S}$ containing $s$, which exists after possibly replacing $\mathcal{S}$ by a dense open subscheme, and set $\mathcal{X}^{\prime}=\mathcal{X} \times \mathcal{S} \mathcal{S}^{\prime}$. By the induction assumption there is a $\xi^{\prime} \in K_{0}\left(\mathcal{X}^{\prime}\right)_{\mathbb{Q}}$ with $\operatorname{ch}\left(\left.\xi^{\prime}\right|_{\mathcal{X}_{s}}\right)=\operatorname{ch}\left(\xi_{s}\right)$.

Let $s^{\prime}$ be the generic point of $\mathcal{S}^{\prime}$ and choose an extension of fields $k \subset k^{\prime} \subset k\left(s^{\prime}\right)$ such that the second inclusion is finite and such that there exists a lift $k^{\prime} \rightarrow \mathcal{O}_{\mathcal{S}, s^{\prime}}$. This lift gives rise to a curve $\mathcal{S}^{\prime \prime} / k^{\prime}$ mapping to $\mathcal{S}$ as schemes over $k$ such that $s^{\prime}$ is contained in the image. Now one applies the one-dimensional case of Conjecture A.1 to the family

$$
\mathcal{X}^{\prime \prime}=\mathcal{X} \times \mathcal{S} \mathcal{S}^{\prime \prime} \rightarrow \mathcal{S}^{\prime \prime} \text { with class }\left.\xi^{\prime}\right|_{s^{\prime}} \in K_{0}\left(\mathcal{X}_{s^{\prime}}\right)_{\mathbb{Q}}
$$

to get a lifted class $\xi^{\prime \prime} \in K_{0}\left(\mathcal{X}^{\prime \prime}\right)_{\mathbb{Q}}$. Finally, $\mathcal{X}^{\prime \prime} \subset \mathcal{X}$ is an inverse limit of open immersions of regular schemes, so we can extend $\xi^{\prime \prime}$ to a class $\xi \in K_{0}(\mathcal{X})_{\mathbb{Q}}$, which will then satisfy the requested Conjecture A.1(ii).

Now we assume that $\operatorname{dim}(\mathcal{S})=1$. Without loss of generality, we assume that $k=k(s)$. Using Deligne's partie fixe [Del71, 4.1] we can also assume without loss of generality that the lift $\alpha$ of $\operatorname{ch}\left(\xi_{s}\right)$ in Conjecture A.1(i) lies in the image of

$$
\bigoplus_{i} H^{2 i}\left(\mathcal{X}, \Omega_{\mathcal{X} / k}^{\geqslant i}\right) \rightarrow H_{\mathrm{dR}}^{*}(\mathcal{X} / k)
$$

The completion of $\mathcal{O}_{\mathcal{S}, s}$ along the maximal ideal is isomorphic to $k[[t]]$. So define $X=\mathcal{X} \times \mathcal{S} S$, where $S=\operatorname{Spec} k[[t]]$ and apply Conjecture 1.4 to the class $\xi_{1}=\xi_{s}$ and the flat lift $\left.\alpha\right|_{X}$ of $\operatorname{ch}\left(\xi_{1}\right)$ to get a lifted class $\dot{\xi} \in K_{0}(X)_{\mathbb{Q}}$. 


\section{Bloch, Esnault And KerZ}

Denote by $S^{\mathrm{h}}$ the spectrum of the henselization of $\mathcal{O}_{\mathcal{S}, s}$ and by $X^{\mathrm{h}}$ the pullback $\mathcal{X} \times \mathcal{S} S^{\mathrm{h}}$. By Artin approximation [Art69] there is a class $\ddot{\xi} \in K_{0}\left(X^{\mathrm{h}}\right)_{\mathbb{Q}}$ with $\left.\dot{\xi}\right|_{\mathcal{X}_{s}}=\left.\ddot{\xi}\right|_{\mathcal{X}_{s}}$. By a standard transfer argument we get from $\ddot{\xi}$ a class $\xi \in K_{0}(\mathcal{X})_{\mathbb{Q}}$ with the requested property of Conjecture A.1(ii).

Variational Hodge $\Longrightarrow$ Infinitesimal Hodge:

Let $\xi_{1}$ satisfy property (i) of Theorem 1.2. The idea is roughly the following:

(1) reduce to a situation where $X \rightarrow S$ 'extends' to a morphism between complex varieties $\mathcal{X} \rightarrow \mathcal{S}$

(2) use complex Hodge theory in order to show that $\operatorname{ch}\left(\xi_{1}\right)$ extends as a de Rham cohomology class to $H_{\mathrm{dR}}^{*}(\mathcal{X} / \mathbb{C})$ so that we can apply Conjecture A.1.

By a simple reduction we can assume without loss of generality that $k$ contains $\mathbb{C}$. Then as step (1) we find a local subring $R \subset k[[t]]$ with maximal ideal $m$ which is essentially of finite type over $\mathbb{Q}$ and such that $X$ descends to a projective smooth scheme $X_{R}$ over $R$ and such that $\xi_{1}$ descends to a class in $K_{0}\left(X_{R} \otimes R / m\right)_{\mathbb{Q}}$. Such an $R$ exists by the techniques of [EGA4, Sec. IV.8]. By resolution of singularities we can assume without loss of generality that $R$ is regular. Choose a subfield $k^{\prime} \subset R$ such that the field extension $k^{\prime} \subset R / m$ is finite and such that $k^{\prime}$ is algebraically closed in $R$. Now we can extend

$$
\operatorname{Spec}\left(X_{R} \otimes_{k^{\prime}} \mathbb{C}\right) \rightarrow \operatorname{Spec}\left(R \otimes_{k^{\prime}} \mathbb{C}\right)
$$

to a smooth projective morphism $f: \mathcal{X} \rightarrow \mathcal{S}$ of smooth varieties over $\mathbb{C}$. There is a canonical morphism $\gamma: S \rightarrow \mathcal{S}$ over $\mathbb{C}$. In other words we get a Cartesian square

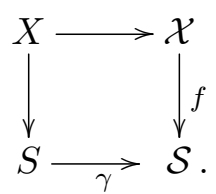

The map $\gamma$ maps the closed point of $S$ to a closed point $s \in \mathcal{S}$ and the generic point of $S$ to the generic point of $\mathcal{S}$. There is an induced class $\xi_{s} \in K_{0}\left(\mathcal{X}_{s}\right)_{\mathbb{Q}}$, which $\gamma$ pulls back to our originally given class $\xi_{1} \in K_{0}\left(X_{1}\right)_{\mathbb{Q}}$.

We claim (step (2)) that the de Rham class $\operatorname{ch}\left(\xi_{s}\right) \in H_{\mathrm{dR}}^{*}\left(\mathcal{X}_{s} / \mathbb{C}\right)$ extends to $H_{\mathrm{dR}}^{*}(\mathcal{X} / \mathbb{C})$ after possibly replacing $\mathcal{S}$ by an étale neighborhood of $s$. This will allow us to apply Conjecture A.1 to obtain a class $\dot{\xi} \in K_{0}(\mathcal{X})_{\mathbb{Q}}$, so that the requested class in $K_{0}(X)_{\mathbb{Q}}$ from Conjecture 1.4(iii) is given by $\xi=\gamma^{*}(\dot{\xi})$. This will finish the proof of Proposition A.2.

To show the claim let $f^{\mathbb{C}}: \mathcal{X}(\mathbb{C}) \rightarrow \mathcal{S}(\mathbb{C})$ be the induced map of complex manifolds and consider the local system $L=R^{*} f_{*}^{\mathbb{C}} \mathbb{Q}$ on $\mathcal{S}(\mathbb{C})$ (we omit any Tate twists). We think of $L$ as an étale manifold over $\mathcal{S}(\mathbb{C})$. In this sense let $L_{0}$ be the connected component of the Betti Chern character class $\operatorname{ch}\left(\xi_{s}\right) \in H_{\mathrm{B}}^{*}\left(\mathcal{X}_{s}(\mathbb{C})\right)$ in the unramified complex space

$$
L \cap \bigoplus_{i \geqslant 0} R^{2 i} f_{*}^{\mathbb{C}}\left(\Omega_{\mathcal{X} / \mathcal{S}}^{\geqslant i}\right)
$$

over $\mathcal{S}(\mathbb{C})$.

By [CDK95] we know that $L_{0}$ is finite over $\mathcal{S}(\mathbb{C})$ and therefore given by a finite unramified scheme over $\mathcal{S}$, which we denote by the same letter. After replacing $\mathcal{S}$ by an étale neighborhood of $s$ we can therefore assume that $L_{0} \rightarrow \mathcal{S}$ is a closed immersion. 


\section{Deformation of CYCle ClASSES}

Clearly, $\gamma^{*}\left(L_{0}\right)$ contains the locus where $\Phi^{-1} \circ \operatorname{ch}\left(\xi_{1}\right)$ lies in the Hodge filtration

$$
\bigoplus_{i \geqslant 0} F^{i} H_{\mathrm{dR}}^{2 i}(X / S)
$$

(the map $\Phi$ is defined in (1.2)). By our assumption on $\xi_{1}$ this locus is all of $S$. So we get that $L_{0} \rightarrow \mathcal{S}$ is an isomorphism, since $\gamma$ has dense image. This means that the monodromy action of $\pi_{1}(\mathcal{S}(\mathbb{C}), s)$ on $H_{\mathrm{B}}^{*}\left(\mathcal{X}_{s}(\mathbb{C})\right)$ fixes $\operatorname{ch}\left(\xi_{s}\right)$. By the degeneration of the Leray spectral sequence [Del71, 4.1] the cohomology class $\operatorname{ch}\left(\xi_{s}\right) \in H_{\mathrm{dR}}^{*}\left(\mathcal{X}_{s} / \mathbb{C}\right)$ extends to $H_{\mathrm{dR}}^{*}(\mathcal{X} / \mathbb{C})$, proving the claim.

\section{Appendix B. A counterexample to algebraization}

In this section, we show that algebraization of $K_{0}$-classes of vector bundles does not hold in general, that is, the map (1.3) is usually 'far' from being an isomorphism. For a precise statement see Proposition B.5. The idea is to consider a 'pro-0-cycle' on the trivial deformation over $\mathbb{C}[[t]]$ of a smooth projective variety $Y / \mathbb{C}$ with $p_{g}>0$. Roughly speaking we construct such a pro-0-cycle whose top Chern class in absolute Hodge cohomology 'jumps' around so much in the pro-system that it cannot come from absolute differential forms on $Y \otimes_{\mathbb{C}} \mathbb{C}[[t]]$.

We start the discussion with certain elementary observations about absolute differential forms. One defines a weight function on differential forms $\tau \in \Omega_{\mathbb{C} / \mathbb{Q}}^{2}$ by setting

$$
w(\tau):=\min \left\{n \mid \tau=\sum_{i=1}^{n} a_{i} d b_{i} \wedge d c_{i}, a_{i}, b_{i}, c_{i} \in \mathbb{C}\right\} .
$$

The function $w$ is subadditive in the sense that

$$
w\left(\tau_{1}+\ldots+\tau_{p}\right) \leqslant \sum w\left(\tau_{i}\right) .
$$

LEMma B.1. Let $\tau=\sum_{i=1}^{n} d b_{i} \wedge d c_{i}$ and assume that all the $b_{i}, c_{i}$ are algebraically independent elements in $\mathbb{C}$. Then $w(\tau)=n$.

Proof. Clearly $w(\tau) \leqslant n$. If $w(\tau)<n$ we can write

$$
\tau=\sum_{j=1}^{n-1} \alpha_{j} d \beta_{j} \wedge d \gamma_{j}
$$

The $n$-fold wedge $\wedge^{n} \tau=\tau \wedge \cdots \wedge \tau \in \Omega_{\mathbb{C}}^{n}$ is equal to $n ! d b_{1} \wedge d c_{1} \wedge d b_{2} \wedge \cdots \wedge d c_{n}$, which is nonzero in $\Omega_{\mathbb{C}}^{2 n}$ as the $a_{i}, b_{j}$ are algebraically independent. On the other hand, if (B.1) holds, then $\wedge^{n} \tau=0$ in $\Omega_{\mathbb{C}}^{2 n}$, a contradiction.

Let $R=\mathbb{C}[[t]]$ and write $R_{n}=R / t^{n} R$.

The choice of a parameter $t$ yields a natural splitting

$$
\Omega_{\mathbb{C}}^{i} \otimes_{\mathbb{C}} R_{n} \rightarrow \Omega_{R_{n}}^{i} \rightarrow \Omega_{\mathbb{C}}^{i} \otimes_{\mathbb{C}} R_{n}
$$

which is compatible in the pro-system in $n$. Thus it defines a homomorphism

$$
\begin{gathered}
\Omega_{R}^{2} \rightarrow \underset{n}{\lim _{n}} \Omega_{\mathbb{C}}^{2} \otimes_{\mathbb{C}} R_{n}=: \Omega_{\mathbb{C}}^{2} \widehat{\otimes} R \\
\gamma=\sum_{i=1}^{N} f_{i}(t) d g_{i}(t) \wedge d h_{i}(t) \mapsto \tilde{\gamma}=\sum_{p=1}^{\infty} t^{p} \tau_{p}, \\
f_{i}, g_{i}, h_{i} \in R, \tau_{p} \in \Omega_{\mathbb{C}}^{2} .
\end{gathered}
$$




\section{Bloch, Esnault and Kerz}

Lemma B.2. One has $w\left(\tau_{p}\right) \leqslant N\left(\begin{array}{c}p+2 \\ p\end{array}\right)$.

Proof. Suppose first $N=1$ and $\omega=f d g \wedge d h$. Write $f=\sum_{j=0}^{\infty} f^{(j)} t^{j}$ and similarly for $g$, $h$. Then

$$
f d g \wedge d h=\sum_{p} t^{p} \sum_{i+j+k=p} f^{(i)} d g^{(j)} \wedge d h^{(k)} .
$$

The inner sum has $\left(\begin{array}{c}p+2 \\ p\end{array}\right)$ terms, and the result follows in this case simply by the definition of $w$. For $N$ general, we conclude by the subadditivity of $w$.

Lemma B.3. Let

$$
\tilde{\eta}=\sum_{p=0}^{\infty} t^{p} \eta_{p} \in \Omega_{\mathbb{C}}^{2} \widehat{\otimes} \mathbb{C}[[t]] .
$$

Assume that

$$
\limsup _{p \rightarrow \infty} \frac{w\left(\eta_{p}\right)}{p^{2}}=\infty
$$

Then $\tilde{\eta}$ does not lift via (B.3) to an element in $\Omega_{\mathbb{C}[[t]]}^{2}$.

Proof. Immediate from Lemma B.2.

Remark B.4. Lemmas B.1-B.3 immediately generalize to differential forms of any even degree.

Let $Y / \mathbb{Q}$ be a smooth projective variety. We write $Y_{A}$ for the base change by a $\operatorname{ring} A / \mathbb{Q}$.

Proposition B.5. Assume that $p_{g}=\operatorname{dim}_{\mathbb{Q}} H^{0}\left(Y, \Omega_{Y}^{\operatorname{dim} Y}\right)>0$ and that the dimension of $Y$ is even. Then the map

$$
K_{0}\left(Y_{R}\right)_{\mathbb{Q}} \rightarrow(\underbrace{}_{n} K_{0}\left(Y \times_{\mathbb{C}} R_{n}\right)) \otimes \mathbb{Q}
$$

is not surjective.

For simplicity of notation we restrict ourselves to $\operatorname{dim} Y=2$ for the rest of this section. The proof of the general case of Proposition B.5 works exactly the same way. For $A$ a ring over $\overline{\mathbb{Q}}$, we have a second Chern character in absolute Hodge cohomology

$$
\operatorname{ch}_{2}: K_{0}\left(Y_{A}\right) \rightarrow H^{2}\left(Y_{A}, \Omega_{Y_{A}}^{2}\right) .
$$

Using the Künneth decomposition for differential forms and the resulting projection

$$
H^{2}\left(Y, \Omega_{Y_{A}}^{2}\right) \rightarrow H^{2}\left(Y, \mathcal{O}_{Y}\right) \otimes \Omega_{A}^{2}
$$

one defines by composition

$$
\widetilde{c h}_{2}: K_{0}\left(Y_{A}\right) \rightarrow H^{2}\left(Y_{A}, \Omega_{Y_{A}}^{2}\right) \rightarrow H^{2}\left(Y, \mathcal{O}_{Y}\right) \otimes \Omega_{A}^{2} .
$$

Taking above $A$ to be $R_{n}$ and composing with the projection in (B.2), one obtains the homomorphism

$$
\overline{\operatorname{ch}}_{2}: K_{0}\left(Y_{R_{n}}\right) \rightarrow H^{2}\left(Y, \mathcal{O}_{Y}\right) \otimes_{\overline{\mathbb{Q}}} \Omega_{\mathbb{C}}^{2} \otimes_{\mathbb{C}} R_{n}
$$

For the following discussion we choose a point $z \in Y(\overline{\mathbb{Q}})$ and generators $t_{1}, t_{2}$ of the maximal ideal of $\mathcal{O}_{Y, z}$. Write $X=\operatorname{Spec} \mathcal{O}_{Y, z}$ and $U=X \backslash z$. This choice gives rise to an element $\rho \in$ $H^{2}\left(Y, \mathcal{O}_{Y}\right)$ by the following construction. In fact for later reference we explain the construction after performing a base change to $\mathbb{C}$. Consider the covering $\mathcal{U}=\left(U_{i}\right)_{i=1,2}$ of $U_{\mathbb{C}}$ with $U_{i}=$ 


\section{Deformation of CyCle Classes}

$X_{\mathbb{C}} \backslash V\left(t_{i}\right)$. Now $\rho$ is the image of the Čech cocycle $1 / t_{1} t_{2} \in \check{H}^{1}\left(\mathcal{U}, \mathcal{O}_{U_{\mathbb{C}}}\right)$ under the composite map

$$
\begin{aligned}
H_{n}^{r}: \check{H}^{1}\left(\mathcal{U}, \Omega_{U_{R_{n}}}^{r}\right) \rightarrow & H^{1}\left(U_{\mathbb{C}}, \Omega_{U_{R_{n}}}^{r}\right) \rightarrow H_{z}^{2}\left(X_{\mathbb{C}}, \Omega_{X_{R_{n}}}^{r}\right) \\
& \sim H_{z}^{2}\left(Y_{\mathbb{C}}, \Omega_{Y_{R_{n}}}^{r}\right) \rightarrow H^{2}\left(Y_{\mathbb{C}}, \Omega_{Y_{R_{n}}}^{r}\right)
\end{aligned}
$$

for $n=1$ and $r=0$.

Lemma B.6. Assume that $p_{g}=\operatorname{dim}_{\overline{\mathbb{Q}}} H^{0}\left(Y, \Omega^{2}\right)>0$. Then a generic choice of $z$ gives rise to nonvanishing $\rho \in H^{2}\left(Y, \mathcal{O}_{Y}\right)$.

Proof. Choose a nonvanishing $\omega \in H^{0}\left(Y, \Omega_{Y}^{2}\right)$. Duality theory [Har66] shows that $\omega \cup \rho \in$ $H^{2}\left(Y, \Omega_{Y}^{2}\right) \cong \overline{\mathbb{Q}}$ does not vanish if $\omega$ does not vanish at $z$.

Lemma B.7. For $n \geqslant 1$, the image of

$$
\begin{aligned}
\operatorname{ker}\left(K_{0}\left(Y_{R_{n+1}}\right) \rightarrow\right. & \left.K_{0}\left(Y_{R_{n}}\right)\right) \cap \operatorname{im}\left(K_{0}\left(Y_{R}\right) \rightarrow K_{0}\left(Y_{R_{n+1}}\right)\right) \\
& \stackrel{\overline{c h}_{2}}{\longrightarrow} H^{2}\left(Y, \mathcal{O}_{Y}\right) \otimes_{\overline{\mathbb{Q}}} \Omega_{\mathbb{C}}^{2} \otimes_{\mathbb{C}} R_{n+1}
\end{aligned}
$$

contains any element of the form $\rho \otimes t^{n}\left(d a_{1} \wedge d b_{1}+\cdots+d a_{p} \wedge d b_{p}\right)$ with $a_{i}, b_{i} \in \mathbb{C}$.

Proof. It suffices to show that any element of the form $\rho \otimes t^{n} d a \wedge d b$ lies in the image of the map (B.8).

According to Grothendieck-Riemann-Roch [Ful84, Ex. 15.2.15] one has the following result.

Claim B.8.

(i) For any $R_{n+1}$-point $x \in Y\left(R_{n+1}\right)$ there is a canonical pushforward $\mathbb{Z}=K_{0}\left(R_{n+1}\right) \rightarrow$ $K_{0}\left(Y_{R_{n+1}}\right)$. We denote the image of 1 under this map by $[x]$.

(ii) Assume that $x$ as in (i) lifts the point $z$. Then $\operatorname{ch}_{2}([x])$ is equal to

$$
H_{n+1}^{2}\left(d \log \left(t_{1}-x^{*}\left(t_{1}\right)\right) \wedge d \log \left(t_{2}-x^{*}\left(t_{2}\right)\right)\right),
$$

where the map $H_{n+1}^{2}$ is as defined in (B.7). by

For $a, b \in \mathbb{C}$ we consider two $R_{n+1}$-points $x, y \in Y\left(R_{n+1}\right)$ specializing to $z$ which are defined

$$
\begin{gathered}
x: t_{1} \mapsto a t^{n-1}, t_{2} \mapsto b t, \\
y: t_{1} \mapsto 0, t_{2} \mapsto b t .
\end{gathered}
$$

Observe that the pullbacks of $[x],[y]$ to $K_{0}\left(X_{R_{n}}\right)$ coincide. It is clear that $x$ and $y$ extend to $R$-points of $Y$. So $[x]-[y]$ lies in the group on the left side of (B.8). The following claim shows that

$$
\overline{\operatorname{ch}}_{2}([x]-[y])=\rho \otimes t^{n} d a \wedge d b,
$$

finishing the proof of Lemma B.7.

Claim B.9.

$$
\begin{gathered}
\overline{\operatorname{ch}}_{2}([x])=\rho \otimes t^{n} d a \wedge d b \\
\overline{\operatorname{ch}}_{2}([y])=0 .
\end{gathered}
$$




\section{Bloch, Esnault and Kerz}

Proof. We give the proof for $x$, the case of $y$ works similarly. In the Čech cohomology group $\check{H}^{2}\left(\mathcal{U}, \mathcal{O}_{U}\right) \otimes_{\overline{\mathbb{Q}}} \Omega_{\mathbb{C}}^{2} \otimes_{\mathbb{C}} R_{n+1}$ we have

$$
\begin{aligned}
d \log \left(t_{1}-x^{*}\left(t_{1}\right)\right) \wedge d \log \left(t_{2}-x^{*}\left(t_{2}\right)\right) & =\frac{d x^{*}\left(t_{1}\right) \wedge d x^{*}\left(t_{2}\right)}{\left(t_{1}-x^{*}\left(t_{1}\right)\right)\left(t_{2}-x^{*}\left(t_{2}\right)\right)} \\
& =\frac{t^{n} d a \wedge d b}{\left(t_{1}-x^{*}\left(t_{1}\right)\right)\left(t_{2}-x^{*}\left(t_{2}\right)\right)}=\frac{t^{n} d a \wedge d b}{t_{1} t_{2}} .
\end{aligned}
$$

So (B.11) follows from Claim B.8(ii) and the definition of $\rho$.

Let $K=\mathbb{C}((t))$.

Lemma B.10. One has $K_{0}\left(Y_{R}\right) \cong K_{0}\left(Y_{K}\right)$.

Proof. The boundary $\partial: K_{1}\left(Y_{K}\right) \rightarrow K_{0}\left(Y_{k}\right)$ is surjective: as $Y_{R}$ admits a morphism $Y_{R} \rightarrow Y_{k}$, one applies the formula $x=\partial\left(x_{R} \cdot[t]\right)$ where $x_{R} \in K_{0}\left(Y_{R}\right)$ is the pullback of $x \in K_{0}\left(Y_{k}\right)$ via the projection $Y_{R} \rightarrow X_{k}$ and $[t] \in K_{1}(K)$ is the class of the unit $t \in K^{\times}$. It follows that $K_{0}\left(Y_{R}\right) \hookrightarrow K_{0}\left(Y_{K}\right)$. For the surjectivity, it suffices to note that a coherent sheaf on $Y_{K}$ can be extended to a coherent sheaf on $Y_{R}$, and, as $Y_{R}$ is regular, it can be resolved by locally free sheaves.

Proof of Proposition B.5. Recall that for simplicity of notation we assume that $\operatorname{dim} Y=2$. The diagram

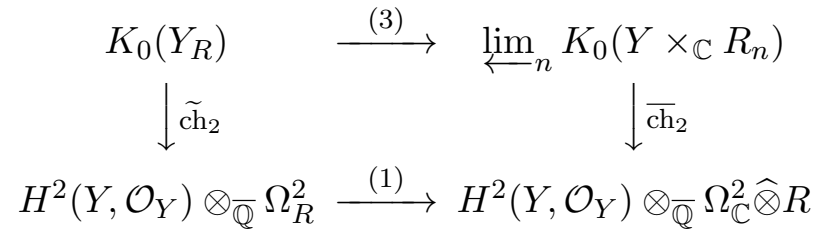

commutes. By Lemma B.7, the image of $\overline{\mathrm{ch}}_{2}$ contains all elements of the form $\rho \otimes \sum_{n=1}^{\infty} t^{n} \tau_{n}$, where $\tau_{n}=\sum_{i=1}^{p(n)} d a_{i}^{(n)} \wedge d b_{i}^{(n)}$. Here all the $a_{i}^{(n)}$ and $b_{i}^{(n)}$ are chosen algebraically independent, and we choose a sequence $\{p(n)\}$ such that $\lim _{\sup _{n}}(p(n) / n)^{2}=\infty$. It follows from Lemma B.3 that $\rho \otimes\left(\sum_{n=1}^{\infty} t^{n} \tau_{n}\right)$ does not lie in the image of (1) if $\rho \neq 0$, so the map labeled (3) cannot be surjective in this case. Note that by Lemma B.6 a generic choice of the point $z \in Y(\overline{\mathbb{Q}})$ gives rise to nonvanishing $\rho$.

Remark B.11. Of course there are also odd-dimensional varieties $X$, for which algebraization fails. Take for example $X=Y \times{ }_{\overline{\mathbb{Q}}} \mathbb{P}^{1}$ with $Y$ as in Proposition B.5. In fact any smooth projective $X / \overline{\mathbb{Q}}$ which maps surjectively onto such a $Y$ does not satisfy algebraization in the sense of Proposition B.5.

\section{ACKNOWLEDGEMENTS}

We would like to thank A. Beilinson for many comments on our work on the deformation of cycles.

\section{REFERENCES}

EGA3 A. Grothendieck and J. Dieudonné, Éléments de géométrie algébrique III: Étude cohomologique des faisceaux cohérents, Inst. Hautes Études Sci. Publ. Math. 11 (1961), 17 (1963). 


\section{DEFORMATION OF CYCle ClASSES}

EGA4 A. Grothendieck and J. Dieudonné, Éléments de géométrie algébrique IV: Étude locale des schémas et des morphismes de schémas, Inst. Hautes Études Sci. Publ. Math. 20, 24, 28, 32 (1964).

Abd94 S. Abdulali, Algebraic cycles in families of abelian varieties, Canad. J. Math. 46 (1994), no. 6, 1121-1134. http://dx.doi.org/10.4153/CJM-1994-063-0

And96 Y. André, Pour une théorie inconditionnelle des motifs, Inst. Hautes Études Sci. Publ. Math. 83 (1996), 5-49. http://dx.doi.org/10.1007/BF02698643

Art69 M. Artin, Algebraic approximation of structures over complete local rings, Inst. Hautes Études Sci. Publ. Math. 36 (1969), 23-58.

Bas68 H. Bass, Algebraic K-theory, W. A. Benjamin, Inc., New York-Amsterdam 1968.

Blo72 S. Bloch, Semi-regularity and de Rham cohomology, Invent. Math. 17 (1972), 51-66.

Blo75 S. Bloch, $K_{2}$ of Artinian $\mathbb{Q}$-algebras, with application to algebraic cycles, Comm. Algebra 3 (1975), 405-428.

Blo86 S. Bloch, Algebraic Cycles and Higher K-Theory, Adv. in Math. 61 (1986), 267-304.

BO75 S. Bloch and A. Ogus, Gersten's conjecture and the homology of schemes, Ann. Sci. École Norm. Sup. 7 (1975), 181-201.

CDK95 E. Cattani, P. Deligne and A. Kaplan, On the locus of Hodge classes, J. Amer. Math. Soc. 8 (1995), no. 2, 483-506. http://dx.doi.org/10.2307/2152824

Del71 P. Deligne, Théorie de Hodge II, Inst. Hautes Études Sci. Publ. Math. 40 (1971), 5-57.

DM91 C. Deninger and J. Murre, Motivic decomposition of abelian schemes and the Fourier transform, J. Reine Angew. Math. 422 (1991), 201-219.

FL85 W. Fulton and S. Lang, Riemann-Roch algebra, Springer-Verlag, New York, 1985. http://dx. doi.org/10.1007/978-1-4757-1858-4

Ful84 W. Fulton, Intersection Theory, Ergebnisse der Mathematik und ihrer Grenzgebiete, 3. Folge, Bd. 2, Springer-Verlag, Berlin, 1984. http://dx.doi.org/10.1007/978-3-662-02421-8

Gil81 H. Gillet, Riemann-Roch Theorems for Higher K-Theory, Adv. in Math. 40 (1981), 203-289. http://dx.doi.org/10.1016/S0001-8708(81)80006-0

Goo86 T. Goodwillie, Relative algebraic K-theory and cyclic homology, Annals of Math. (2) 124 (1986), no. 2, 347-402. http://dx.doi.org/10.2307/1971283

GG04 M. Green and P. Griffiths, Formal deformation of Chow groups, The Legacy of Niels Henrik Abel, 467-509, Springer-Verlag, Berlin, 2004. http://dx. doi.org/10.1007/978-3-642-18908-1_13

Gro66 A. Grothendieck, On the de Rham cohomology of algebraic varieties, Inst. Hautes Études Sci. Publ. Math. 29 (1966), 95-103.

Har66 R. Hartshorne, Residues and duality, Lecture notes of a seminar on the work of A. Grothendieck, given at Harvard 1963/64, Lecture Notes in Mathematics, vol. 20, Springer-Verlag, Berlin-New York, 1966; with an appendix by P. Deligne.

Isa04 D. Isaksen, Strict model structures for pro-categories, Categorical decomposition techniques in algebraic topology (Isle of Skye, 2001), Progr. Math., 215, Birkhäuser, Basel, 2004, 179-198. http://dx.doi.org/10.1007/978-3-0348-7863-0_11

Jan94 U. Jannsen, Motivic sheaves and filtrations on Chow groups, Motives (Seattle, WA, 1991), Amer. Math. Soc., Providence, RI, 1994, 245-302. http://dx.doi.org/10.1090/pspum/055. $1 / 1265533$

Kat70 N. Katz, Nilpotent connections and the monodromy theorem: Applications of a result of Turrittin, Inst. Hautes Études Sci. Publ. Math. 39 (1970), 175-232.

Ker09 M. Kerz, The Gersten conjecture for Milnor K-theory, Invent. Math. 175 (2009), 1-33. http: //dx.doi.org/10.1007/s00222-008-0144-8

MVW06 C. Mazza, V. Voevodsky and C. Weibel, Lecture notes on motivic cohomology, Clay Mathematics Monographs, 2, Amer. Math. Soc., Providence, RI, 2006. 


\section{Bloch, Esnault and Kerz}

Mor13a M. Morrow, Deformational Hodge conjecture versus Pro HKR, C. R. Math. Acad. Sci. Paris 352 (2014), no. 3, 173-177.

Mor13b M. Morrow, A case of the deformational Hodge conjecture via a pro Hochschild-KostantRosenberg theorem, Preprint (2013), available at http://www.math.uni-bonn.de/people/ morrow/

PR13 D. Patel, and G. V. Ravindra, Weak Lefschetz for Chow groups: infinitesimal lifting, Preprint (2013), available at http://www.ihes.fr/ patel/

Ros96 M. Rost, Chow groups with coefficients, Doc. Math. 1 (1996), no. 16, 319-393.

TT90 R. Thomason and T. Trobaugh, Higher algebraic $K$-theory of schemes and of derived categories, The Grothendieck Festschrift, Vol. III, Progress Math. 88, Birkhäuser Boston, Boston, MA, 1990, 247-435. http://dx.doi.org/10.1007/978-0-8176-4576-2_10

Wei94 C. Weibel, An introduction to homological algebra, Cambridge Studies in Advanced Mathematics, 38, Cambridge University Press, Cambridge, 1994. http://dx.doi.org/10.1017/ CB09781139644136

Spencer Bloch spencer_bloch@yahoo.com

5765 S. Blackstone Ave., Chicago, IL 60637, USA

Hélène Esnault esnault@math.fu-berlin.de

FU Berlin, Mathematik, Arnimallee 3, 14195 Berlin, Germany

Moritz Kerz moritz.kerz@mathematik.uni-regensburg.de

Fakultät für Mathematik, Universität Regensburg, 93040 Regensburg, Germany 